Cite as: P. S. Heeger et al., Sci. Immunol. 10.1126/sciimmunol.abj6513 (2021).

\title{
CORONAVIRUS
}

\section{Implications of defective immune responses in SARS-CoV-2 vaccinated organ transplant recipients}

\author{
Peter S. Heeger ${ }^{1 *}$, Christian P. Larsen ${ }^{2 *}$, Dorry L. Segev ${ }^{3 * \dagger}$ \\ ${ }^{1}$ Translational Transplant Research Center, Icahn School of Medicine at Mount Sinai, New York, NY. ${ }^{2}$ Emory Transplant Center, Emory University School of Medicine, Atlanta, \\ GA. ${ }^{3}$ Transplant Surgery, Johns Hopkins University School of Medicine, Baltimore, MD. \\ *All authors contributed equally. \\ *Corresponding author. Email: dorry@jhmi.edu
}

Organ transplant patients have poor immune responses to COVID-19 vaccines; thus designing vaccine strategies to protect this vulnerable population from SARS-CoV-2 infection is crucial.

Effective vaccines against viruses, including vaccines against SARS-CoV-2, induce virus-specific immune memory comprised of cellular and humoral components that together prevent infection following exposure to the virus; however, many of these responses are compromised in solid organ transplant patients. Initial $\mathrm{T}$ cell and $\mathrm{B}$ cell activation in response to any immune stimuli, including vaccines, requires innate immune activation signals commonly transmitted by pathogen associated molecular patterns ligating pattern recognition receptors expressed on various immune cells. Subsequently, vaccine-induced protective humoral immunity requires collaborative interactions between subsets of CD4+ $\mathrm{T}$ cells, including follicular helper $\mathrm{T}$ cells $\left(\mathrm{T}_{\mathrm{FH}}\right)$, and activated $\mathrm{B}$ cells within germinal centers (GC), a process that results in production of memory CD4+ $\mathrm{T}$ cell populations as well as high affinity, memory $\mathrm{B}$ cells and long-lived, antibodysecreting plasma cells. In addition, effective vaccines induce expansion and differentiation of antigen-specific, memory CD8+ T cells which can rapidly develop into effector cytotoxic lymphocytes capable of killing virus infected cells. Emerging evidence indicates that differentiation of memory CD8+ T cells proceeds through requisite intermediaries including a highly proliferative stem-like memory CD8+ progenitor cell. Key features that distinguish immune memory from the naïve state include a) elevated frequencies of antigen specific $\mathrm{T}$ cells, B cells and antibody secreting cells, b) increased antibody affinity for target antigen, and c) the ability to more rapidly respond to the inciting stimulus. Multiple vaccine doses can boost the primary response induced by the initial vaccination by providing supplementary innate immune activation signals, promoting further expansion of previously $\mathrm{T}$ - and $\mathrm{B}$ cell clones activated by the initial vaccine, and by promoting GC responses that further enhance antibody affinity maturation and antibody production.

Inherited or acquired defects in any of these interrelated immunological processes could potentially disrupt development of protective immunity in response to the vaccine, especially the SARS-CoV-2 vaccines. In a recent issue of Science Immunology, Rincon-Arevalo and colleagues add to the emerging literature documenting that one specific at-risk population for SARS-CoV-2 vaccine failure is organ transplant recipients (1). Transplant recipients require life-long immunosuppression regimens, which commonly include some combination of a calcineurin inhibitor (such as tacrolimus), a steroid, and/or an anti-metabolite (such as mycophenolic acid or mycophenolate mofetil (MMF)). These regimens nonspecifically inhibit $\mathrm{T}$ and $\mathrm{B}$ cells to prevent rejection of the transplanted organ, but also have well-documented and anticipated off-target effects including an increased risk of infection and malignancies. Several publications from 2021 have now documented that immunosuppressed transplant recipients do not routinely develop protective antibody titers following full vaccination with any of the approved SARSCoV-2 mRNA (2) or viral vector (3) vaccines. Associative evidence suggests that use of MMF, an anti-proliferative agent that affects $\mathrm{T}$ and $\mathrm{B}$ cells, contributes to the lack of response $(1,2)$. A more detailed understanding of the immunosuppression-induced defects following SARS-CoV-2 vaccination is needed in order to guide new approaches to induce protective immunity in this at-risk population.

Expanding upon published data, Rincon-Arevalo and colleagues show that absence of protective IgG in vaccinated kidney transplant recipients (KTR) associates with defects in frequencies of RBD-specific, class switched B cells, circulating plasmablasts, and proliferating lymphocyte (including $T C F 7+C D 27+G Z M K+$ stem like CD8+ T cell memory precursor) subsets compared to healthy controls (1). These observations suggest that multiple components of the immune response required for protective antibody production are impaired in KTRs after vaccination against SARS-CoV-2. 
Analogous analyses of dialysis patients revealed delayed, but not absent, protective immunity following vaccination, supporting the conclusion that the immunosuppression (rather than the kidney disease) is likely the etiology of the observed defects in the KTRs. While the authors did not specifically analyze RBD-specific T cells in any of the subjects, the diminished numbers of proliferating CD8+ T cells suggest that KTR may not develop protective $\mathrm{T}$ cell immunity following vaccination.

These disappointing findings have significant clinical implications on two levels. At the individual level, current methods may not achieve adequate protection for those on immunosuppression; this concern is supported by emerging reports (4) of a higher-than-expected rate of breakthrough SARS-CoV-2 infections (including the highly infectious delta variant) in transplant patients. As such, transplant patients must sustain infection control measures and medical treatment must continue to rely on passive immune antibody transfer until effective vaccine regimens are developed. While antibody testing is commercially available, correlates of protective immunity remain unknown in both immunocompetent individuals as well as those taking immunosuppression (with likely suboptimal $\mathrm{T}$ cell responses as well), so all transplant recipients, regardless of antibody levels, should be counseled to "act unvaccinated" until protective immunity is better understood. At the population level, transplant recipients who have failed to achieve adequate protection may create a reservoir for continued viral spread and importantly for ongoing mutation; with around 500,000 transplant patients alive in the United States today, and many more worldwide, this could have a substantial impact on public health.

These findings also help prioritize the next wave of research and development of SARS-CoV-2 vaccination strategies in transplant recipients. While antibody levels are emerging as correlates of protection in healthy subjects, there is still an urgent need to understand the relative contribution of humoral and $\mathrm{T}$ cell immunity in conferring protection. In particular, it is important to understand if the CD8+ T cell response in absence of antibody confers full, partial, or no protection in transplant patients who have received a SARSCoV-2 vaccine. Validated biomarkers of protection are also needed in both immunocompetent and immunosuppressed populations. A more granular understanding beyond antibodies is crucial for individual assessment and clinical decision-making, and also for assessment of trials seeking to confer protection against SARS-CoV-2 in transplant patients and other immunosuppressed populations.

While the immunology literature supports efficacy of booster vaccines and booster doses of SARS-CoV-2 vaccines have been reported to augment protective antibodies in a handful of transplant patients (5), it is important to emphasize that these approaches have not been tested rigorously in clinical trials, the impact of boosters on $\mathrm{T}$ cell immunity has not been determined, and that this relatively simple strategy was not successful in all patients. Additional boosters, boosters of another class of vaccine, or higher dose boosters may rescue more patients, although others may require immunosuppression modulation to develop full protective responses. These escalating approaches entail increasing risk of $T$ cell or antibody mediated injury to the allograft which may not be reversible. Hence clinical trials performed in controlled settings with careful allograft monitoring are required; these trials should be rigorous but rapid, and adaptive methodology might help with efficiency.

The global scientific response to the SARS-CoV-2 pandemic has been a marvel, from the identification of an unknown virus, the development of new diagnostics, immune monitoring and vaccines, to unprecedented global mass vaccination within a year. But vulnerable populations remain, and there is no time to rest in the last mile. The full force of scientific effort is still needed to provide immune protection for those who remain at risk from this deadly virus and to control the reservoir to prevent fertile fields for mutations that put the entire population at risk.

\section{REFERENCES AND NOTES}

1. H. Rincon-Arevalo, M. Choi, A. L. Stefanski, F. Halleck, U. Weber, F. Szelinski, B. Jahrsdörfer, H. Schrezenmeier, C. Ludwig, A. Sattler, K. Kotsch, A. Potekhin, Y. Chen, G. R. Burmester, K. U. Eckardt, G. M. Guerra, P. Durek, F. Heinrich, M. Ferreira-Gomes, A. Radbruch, K. Budde, A. C. Lino, M. F. Mashreghi, E. Schrezenmeier, T. Dörner, Impaired humoral immunity to SARS-CoV-2 BNT162b2 vaccine in kidney transplant recipients and dialysis patients. Sci. Immunol. 6, eabj1031 (2021). doj:10.1126/sciimmunol.abj1031 Medline

2. B. J. Boyarsky, W. A. Werbel, R. K. Avery, A. A. R. Tobian, A. B. Massie, D. L. Segev, J. M. Garonzik-Wang, Antibody Response to 2-Dose SARS-CoV-2 mRNA Vaccine Series in Solid Organ Transplant Recipients. JAMA 325, 2204-2206 (2021). doi:10.1001/jama.2021.7489 Medline

3. B. J. Boyarsky, T. P. Chiang, M. T. Ou, W. A. Werbel, A. B. Massie, D. L. Segev, J. M. Garonzik-Wang, Antibody Response to the Janssen COVID-19 Vaccine in Solid Organ Transplant Recipients. Transplantation (2021). doi:10.1097/TP.0000000000003850 Medline

4. N. M. Ali, N. Alnazari, S. A. Mehta, B. Boyarsky, R. K. Avery, D. L. Segev, R. A. Montgomery, Z. A. Stewart, Development of COVID-19 Infection in Transplant Recipients After SARS-CoV-2 Vaccination. Transplantation (2021). doi:10.1097/TP.0000000000003836 Medline

5. W. A. Werbel, B. J. Boyarsky, M. T. Ou, A. B. Massie, A. A. R. Tobian, J. M. GaronzikWang, D. L. Segev, Safety and Immunogenicity of a Third Dose of SARS-CoV-2 Vaccine in Solid Organ Transplant Recipients: A Case Series. Ann. Intern. Med. (2021). doi:10.7326/L21-0282 Medline

Acknowledgments: Funding: CPL is supported by NIH NIAID U01 Al138909 and the James M. Cox Foundation. PSH is supported by NIH NIAID U01 AI136816. DS is supported by NIH NIAID K24AI144954, U01AI134591, and U01AI138897, and the Ben Dov family. Conflicts of interest: DS reports serving as a consultant to and/or receiving honoraria for speaking from Sanofi, Novartis, CSL Behring, Jazz Pharmaceuticals, Veloxis, Mallinckrodt, and Thermo Fisher Scientific. Author contributions: All authors contributed equally to this manuscript.

Published First Release 1 July 2021

10.1126/sciimmunol.abj6513 